\title{
A Numerical Study on Hydrodynamics of Pectoral Fin Locomotion in Batoid Fishes
}

\author{
W. R. Hu* \\ Department of Engineering Mechanics, Shanghai Jiaotong University, Shanghai 200240, China \\ Email: wrhu@sjtu.edu.cn
}

\begin{abstract}
The mechanics of aquatic locomotion are of interest to biologists, dynamicists and engineers. Batoid fishes(skates and rays) propel themselves through the water primarily with their greatly expanded pectoral fins(pectoral-fin-based locomotion). Batoids exhibit two modes of pectoral swiminng behavior: (1) undulatory locomotion, termed 'rajiform', and (2) oscillatory locomotion, termed 'mobuliform'. A computational study on the unsteady hydrodynamics of pectoral fin locomotion of Rhinoptera Bonasus and Dasyatis Sabina is carried out and presented, which represent the undulatory and oscillatory locomotion, respectively. Unsteady hydrodynamics around a pectoral fin is solved by a time-accurate solution of incompressible, laminar Navier-Stokes equations. Kinematic data of the pectoral fin locomotion used in the computational modeling are based on the experimental results. The pressure distribution of the pectoral fin was computed and integrated to give forces which were decomposed into lift and thrust. The velocity and vorticity field variation on the surface of pectoral fins and in the near-wake was computed throughout the swimming cycle. In the present study, we analyzed and compared the hydrodynamics and mechanmism of the Batoid pectoral fin locomotion between the two modes, and discovered how these patterns change with controllable factors, such as Renolds number, frequency, amplitude etc. The results show that forces on the fins of Rhinoptera Bonasus are much larger than that of Dasyatis Sabina. The load-bearing areas of Rhinoptera Bonasus are at the areas from the leading edge to the medial of the wing; while the load-bearing area of Dasyatis Sabina is the whole wavy fin. These characters are associated with the morphology of the wing skeleton. The propulsive mechanism of pectoral-fin-based locomotion is similar to that of the caudal-fin-based locomotion. A strong backward jet-stream in the wake contributes the net thrust, which is induced by the reversed von Kármán vortex street. The effect of controllable factors on the hydrodynamics in Rhinoptera Bonasus swimming are similar to that in Dasyatis Sabina swimming.
\end{abstract}

\section{REFERENCES}

1. Rosenberger L J. Pectoral fin locomotion in batoid fishes undulation versus oscillation. $J$ Exp Biol, 2001; 204: 379-394

2. Schaefer J T, Summers A P. Batoid wing skeletal structure: novel morphologies, mechanical implications, and phylogenetic patterns. J Morphology, 2005; 264: 298-313 